PROCEEDINGS OF THE

AMERICAN MATHEMATICAL SOCIETY

Volume 123, Number 5, May 1995

\title{
NUMERICAL RADIUS PERSERVING OPERATORS ON $B(H)$
}

\author{
JOR-TING CHAN
}

(Communicated by Palle E. T. Jorgensen)

\begin{abstract}
Let $H$ be a Hilbert space over $\mathbb{C}$ and let $B(H)$ denote the vector space of all bounded linear operators on $H$. We prove that a linear isomorphism $T: B(H) \rightarrow B(H)$ is numerical radius-preserving if and only if it a multiply of a $C^{*}$-isomorphism by a scalar of modulus one.
\end{abstract}

\section{INTRODUCTION}

Let $H$ be a Hilbert space over $\mathbb{C}$ and let $B(H)$ denote the vector space of all bounded linear operators on $H$. For every $A$ in $B(H)$, the numerical range and the numerical radius of $T$ are defined respectively by

$$
\begin{aligned}
W(A) & =\{\langle A x, x\rangle: x \in H,\|x\|=1\}, \\
w(A) & =\sup \{|\lambda|: \lambda \in W(A)\} .
\end{aligned}
$$

It is well known that $w(\cdot)$ is a norm on $B(H)$ and that this norm is equivalent to the usual operator norm. (See [4, p. 117].) A classical theorem of Kadison [4, Theorem 7] asserts that every linear isomorphism on $B(H)$ which is isometric with respect to the operator norm is a $C^{*}$-isomorphism followed by left multiplication by a fixed unitary operator. A $C^{*}$-isomorphism is a linear isomorphism of $B(H)$ such that $T\left(A^{*}\right)=T(A)^{*}$ for all $A$ in $B(H)$ and $T\left(A^{n}\right)=T(A)^{n}$ for all selfadjoint $A$ in $B(H)$ and all natural number $n$. A description of $C^{*}$-isomorphisms on $B(H)$ can be obtained. First of all we have from [6, Corollary 11] that a $C^{*}$-isomorphism on $B(H)$ is either a *isomorphism or a *-anti-isomorphism. Suppose that $T$ is an algebra isomorphism on $B(H)$. Then by [3, Theorem 2], there is an invertible operator $V$ on $H$ such that $T(A)=V A V^{-1}$ for all $A$ in $B(H)$. If we also assume that $T\left(A^{*}\right)=T(A)^{*}$ for all $A$ in $B(H)$, then $V A^{*} V^{-1}=\left(V^{-1}\right)^{*} A^{*} V^{*}$ and hence $\left(V^{*} V\right) A^{*}=A^{*}\left(V^{*} V\right)$ for all $A$ in $B(H)$. It follows that $V^{*} V$ is a scalar multiple of the identity operator $I$. Say $V^{*} V=k I$. As $V^{*} V$ is always a positive operator and $k$ cannot be zero, $k>0$. Let $U=\frac{1}{\sqrt{k}} V$. Then $U$ is unitary and $T(A)=U A U^{*}$ for all $A$ in $B(H)$. For a *-anti-isomorphism $T$, it can be shown (e.g., see [5, Remark 2]) that there is a unitary operator $U$ in $B(H)$ such that $T(A)=U A^{t} U^{*}$ for all $A$ in $B(H)$, where $A^{t}$ denotes the transpose

Received by the editors June 15, 1993 and, in revised form, August 2, 1993.

1991 Mathematics Subject Classification. Primary 47B49, 47A12. 
of $A$ relative to a fixed orthonormal basis of $H$. Clearly operators of these two types are $C^{*}$-isomorphisms.

Let us turn to numerical range and numerical radius. Pellegrini $[9$, Theorem 3.1] proved that an operator $T$ on $B(H)$ is a $C^{*}$-isomorphism exactly when $T$ preserves the "numerical range" of each element in $B(H)$. It should be noted that Pellegrini obtained his result in a general Banach algebra, and his definition of numerical range is different from ours. In fact, for each $A$ in $B(H)$, the "numerical range" of $A$ defined by Pellegrini reduces to the closure of $W(A)$. When the underlying space $H$ is finite-dimensional, $W(A)$ is compact and hence the two sets are identical. Despite the discrepancy we still have that $T$ is a $C^{*}$-isomorphism if and only if $W(T(A))=W(A)$ for every $A$ in $B(H)$. For simplicity we shall call an operator $T$ with the latter property numerical range-preserving. Likewise we say that $T$ is numerical radius-preserving if $w(T(A))=w(A)$ for all $A$ in $B(H)$.

In the finite-dimensional situation, the above result was extended by $\mathrm{Li}$. In [1, Theorem 1] he proved that $T$ is numerical radius-preserving if and only if $T$ is a scalar multiple of a $C^{*}$-isomorphism by a complex number of modulus one. It is immediate that if $T$ is numerical range-preserving, then $T$ is numerical radius-preserving and hence the scalar in question is one. In this note we prove that the conclusion of $\mathrm{Li}$ remains valid without the dimension constraint.

\section{RESULTS}

In what follows $T$ denotes a linear isomorphism on $B(H)$ which is numerical radius-preserving on $B(H)$. We shall prove that $T$ maps the identity mapping $I$ to a scalar multiple of $I$. The scalar is necessarily of modulus one. Multiplying by the complex conjugate of the scalar, we get a numerical radiuspreserving operator $T_{1}$ with an additional property that $T_{1}(I)=I$. The result is concluded by showing that $T_{1}$ is a $C^{*}$-isomorphism.

We begin with a lemma which describes scalar multiples of $I$ in terms of numerical radius. Let $\Lambda=\{\lambda \in \mathbb{C}:|\lambda|=1\}$.

Lemma 1. An operator $A \in B(H)$ is a scalar multiple of $I$ if and only if for every $B \in B(H)$, there is $a \lambda \in \Lambda$ such that $w(A+\lambda B)=w(A)+w(B)$.

Proof. It is clear that if $A$ is a scalar multiple of $I$, then $A$ satisfies the condition. For the converse we borrow the idea from $\mathrm{Li}$ and Tsing [2, p. 40]. We first show that elements in $W(A)$ are of constant modulus; it follows then from the convexity of $W(A)([4, \mathrm{p} .113])$ that the set is a singleton. Hence $A$ is a scalar multiple of the identity $I$. Now assume that there is an $x$ in $H,\|x\|=1$, and $|\langle A x, x\rangle|\langle w(A)$. Let $B$ be the orthogonal projection onto the linear span of $x$. Then $w(B)=1$. Fix any $r$ such that $|\langle A x, x\rangle|<r<w(A)$. We can find an $\varepsilon>0$ such that $|\langle A y, y\rangle|<r$ whenever $\|y-x\|<\varepsilon$. In fact $|\langle A y, y\rangle|<r$ if there is a $\lambda \in \Lambda$ such that $\|y-\lambda x\|<\varepsilon$. Suppose that $y \in H,\|y\|=1$, and $\|y-\lambda x\| \geq \varepsilon$ for every $\lambda \in \Lambda$. Then

$$
\varepsilon^{2} \leq\langle y-\lambda x, y-\lambda x\rangle=2-2 \operatorname{Re}\langle y, \lambda x\rangle \text { for every } \lambda \in \Lambda .
$$

It follows that $\langle y, x\rangle \leq 1-\frac{1}{2} \varepsilon^{2}$. Let $k=\min \left\{r+1, w(A)+1-\frac{1}{2} \varepsilon^{2}\right\}$. Then for every $\lambda \in \Lambda$ and $y \in H$ with $\|y\|=1$, we have

$$
\mid\langle(A+\lambda B) y, y|\leq|\langle A y, y\rangle|+|\langle y, x\rangle| \leq k .
$$

Hence $w(A+\lambda B)<w(A)+w(B)$. 
By the above lemma $T(I)=\lambda I$. Clearly we have $\lambda \in \Lambda$. Let $T_{1}=\bar{\lambda} T$. Then $T_{1}(I)=I$. We need the following definitions. By a state on $B(H)$ we mean as usual a bounded linear functional $\rho$ on $B(H)$ such that $\rho(I)=\|\rho\|=1$. The set $S$ of all states is called the state space of $B(H)$. A bounded linear operator $T: B(H) \rightarrow B(H)$ is said to be state-preserving if its adjoint $T^{\prime}$ satisfies $T^{\prime}(S) \subseteq S$. By [9, Theorem 2.3 and Theorem 3.1], $T$ is a $C^{*}$-isomorphism if and only if it is state-preserving. Let $x$ be a unit vector in $H$. The linear functional $\rho_{x}$ given by

$$
\rho_{x}(A)=\langle A x, x\rangle \text { for every } A \in B(H)
$$

is a state of $B(H)$. States of this form are called vector states.

Lemma 2. The operator $T_{1}$ is state-preserving.

Proof. Let $w^{\prime}$ denote the norm in $B(H)^{\prime}$ dual to the numerical radius. Then $w^{\prime}(\rho) \geq\|\rho\|$ for every $\rho$ in $B(H)^{\prime}$. As $T_{1}$ is numerical radius-preserving, $w^{\prime}\left(T_{1}^{\prime}(\rho)\right)=w^{\prime}(\rho)$ for every $\rho$ in $B(H)^{\prime}$. If $\rho_{x}$ is a vector state, then $w^{\prime}\left(\rho_{x}\right)=$ 1 and hence $\left\|T_{1}^{\prime}\left(\rho_{x}\right)\right\| \leq w^{\prime}\left(T_{1}^{\prime}\left(\rho_{x}\right)\right)=1$. But $T_{1}^{\prime}\left(\rho_{x}\right)(I)=\rho_{x}\left(T_{1}(I)\right)=\rho_{x}(I)=$ 1. It follows that $T_{1}^{\prime}\left(\rho_{x}\right)$ is a state of $B(H)$. By [4, Corollary 4.3.10] the state space is the closed convex hull of the vector states in the weak ${ }^{*}$-topology. This together with the fact that $T_{1}^{\prime}$ is continuous in the weak *-topology entail that $T_{1}$ is state-preserving.

By Lemma 1 and Lemma 2, we have proved

Theorem. A linear isomorphism $T$ on $B(H)$ is numerical radius-preserving if and only if $T$ is a multiple of a $C^{*}$-isomorphism by a scalar of modulus one.

In [1] $\mathrm{Li}$ also studied a numerical radius-preserving real-linear operator on the selfadjoint elements in $B(H)$. He proved ([1, Theorem 2]) that such an operator is the restriction of a $C^{*}$-isomorphism on $B(H)$ multiplied by \pm 1 . Let us remark that as the numerical radius and the operator norm coincide on selfadjoint operators, this result can alternatively be deduced from [7, Theorem 2].

\section{REFERENCES}

1. C. K. Li, Linear operators preserving the numerical radius of matrics, Proc. Amer. Math. Soc. 99 (1987), 601-608.

2. C. K. Li and N. K. Tsing, Linear operators that preserve the c-numerical range or radius of matrices, Linear and Multilinear Algebra 23 (1988), 27-46.

3. M. Eidelheit, On isomorphisms of rings of linear operators, Studia Math. 9 (1940), 97-105.

4. P. R. Halmos, A Hilbert space problem book, 2nd ed., Springer-Verlag, New York, 1982.

5. A. A. Jafarian and A. R. Sourour, Spectrum-preserving linear maps, J. Funct. Anal. 66 (1986), 255-261.

6. R. V. Kadison, Isometries of operator algebra, Ann. of Math. (2) 54 (1951), 325-338.

7. A generalized Schwarz inequality and algebraic invariants for operator algebras, Ann. of Math. (2) 56 (1952), 494-503.

8. R. V. Kadison and J. R. Ringrose, Fundamentals of the theory of operator algebras. I, Academic Press, New York, 1983.

9. V. J. Pellegrini, Numerical range preserving operators on a Banach algebra, Studia Math. 54 (1975), 143-147. 